\title{
Plasma cholesterol concentration and extra lipid band in monoclonal gammopathies
}

\author{
YISHAI LEVY \\ M.D. \\ GAD SPIRA \\ Ph.D \\ GERALD J. BROOK \\ M.D.
}

\author{
MichaEL AVIRAM \\ Ph.D. \\ ILANA TATARSKY \\ M.D.
}

ANNA CARTER

M.D.

Lipid Research Unit and Department of Hematology, Rambam Medical Center and Faculty of Medicine, Technion-Israel Institute of Technology, Haifa, Israel

\section{Summary}

Plasma lipids and lipoproteins were studied in 21 patients with benign monoclonal gammopathy, 21 patients with multiple myeloma and seven patients with Waldenström's macroglobulinaemia. Results were compared with those of a control group, age and sex matched. Low plasma cholesterol levels in all three patient groups were associated with low HDLcholesterol concentrations. Apo A-I, but not apo B, was significantly reduced. Sixty per cent of the patients exhibited an extra lipid band on plasma lipoprotein electrophoresis, which could be an immunoglobulin-lipid complex. In these patients plasma and LDL-cholesterol levels were significantly lower than in those patients in whom this band was absent. No correlation was found between the severity of the disease and plasma lipid pattern.

KEY WORDS: benign monoclonal gammopathy, Waldenström's macroglobulinaemia, multiple myeloma, immunoglobulin, lipoproteins.

\section{Introduction}

Lipoprotein abnormalities have long been recognized amongst patients with multiple myeloma (MM), Waldenström's macroglobulinaemia (WM) and benign monoclonal gammopathy (BMG) (Roberts-Thomson et al., 1975; Beaumont and Beaumont, 1977). Different mechanisms underlying the hyperlipidaemia have been suggested: auto-antibodies reacting with lipoproteins and altering their metabolic fate have been described (Cortese et al., 1982; Baudet, Dachet and Beaumont, 1980).

Most reports have indicated the presence of

Reprints from: Dr M. Aviram, Lipid Research Unit, Rambam Medical Center, Haifa 35254, Israel. hyperlipidaemia in patients with monoclonal gammopathy; hypolipidaemia has been demonstrated less, more often associated with an M protein (Riesen et al., 1972).

We have undertaken the present work in order to (1) define the common lipid and apoprotein patterns found amongst patients with various clinical states associated with monoclonal immunoglobulins, (2) differentiate between the lipoprotein pattern associated with definite malignant conditions such as MM and WM and that associated with the benign monoclonal gammopathies, (3) determine whether the type or degree of lipid abnormality is related to tumour burden or is merely dependent on the serum concentration of monoclonal immunoglobulins.

\section{Patients and methods}

Plasma lipids and lipoproteins were studied in 49 patients with monoclonal gammopathy: 21 patients with multiple myeloma, seven with Waldenström's macroglobulinaemia and 21 with benign monoclonal gammopathy. There were 25 males and 24 females between the ages of 48 and 80 years. The diagnosis of benign monoclonal gammopathy and multiple myeloma was established according to generally accepted criteria (Nationai Cancer Institute, 1973; Kyle, 1978). The diagnosis of Waldenström's macroglobulinaemia was based upon criteria which included definitive evidence of a monoclonal immunoglobulin (Ig) $M$ in the serum, bone marrow infiltration by lymphocytic-plasmacytic cells, hepato-splenomegaly with or without lymphadenopathy. Quantitation of immunoglobulins was made by radial immunodiffusion technique (Fahey and McKelvey, 1965).

Patients with multiple myeloma were staged ac- 
cording to a myeloma clinical staging system that correlates well with tumour burden (Durie and Salmon, 1975). Thirty-four patients had never been treated; 21 had BMG, seven indolent myeloma (Alexanian, 1980); two were newly diagnosed symptomatic MM patients and four with WM; six patients had been off therapy for 1-4 years; and nine had not received treatment for at least 6 weeks. None of the patients had a history of thyroid disease or diabetes mellitus, and no one had evidence of hepatic or renal dysfunction. The patients did not receive drugs known to alter lipid metabolism. All patients were ambulatory, and no one was pre-terminal. Forty-nine age-matched healthy volunteers were controls. All patients and control subjects gave their informed consent for the study.

Blood samples were collected in 0.1\% EDTA, following an overnight fast. Plasma cholesterol and triglycerides levels were determined by enzymatic methods on a centrifugal fast analyser (ElectroNucleonics, Gemsaec). The concentration of high density lipoprotein (HDL)-cholesterol was assayed following precipitation with heparin manganese (Burstein, Scholnick and Martin, 1970). Apolipoproteins A-I and B were measured by rocket electroimmunophoresis according to the method of Laurell (1965). Plasma lipoprotein electrophoresis was performed on cellulose acetate.

\section{Results}

Table 1 summarizes the mean values of $M$ protein levels, plasma lipids and apoprotein concentrations. Low levels of cholesterol were found to be the most common finding amongst the patients under study $(P<0.02)$. Low plasma triglycerides were observed amongst patients with WM $(P<0.02)$, and high levels were found in the group of BMG and MM, but the latter findings were not significant in comparison with the control group. HDL-cholesterol was lower in each of the three clinical groups $(P<0.02)$. Apoprotein A-I concentration generally followed HDLcholesterol level. The decrease in cholesterol level was not accompanied by similar pattern in apoprotein B concentraton.

The presence of an extra lipid band on cellulose acetate electrophoresis (Fig. 1) raised the possibility of an immunoglobulin-lipid complex, because plasma lipoprotein electrophoresis revealed that the extra lipid band was identical in its electrophoretic migration to the immunoglobulin (as demonstrated by plasma protein electrophoresis). Patients demonstrating that band had lower cholesterol and triglyceride concentrations than those that did not possess the same band (Table 2). The extra lipid band was found in the density range $d>1.21 \mathrm{~g} / \mathrm{ml}$, and no apoprotein A-I or B could be detected in this fraction by immunodiffusion technique. No consistent alterations were observed concerning HDL-cholesterol and plasma apoprotein B and apoprotein A-I concentrations.

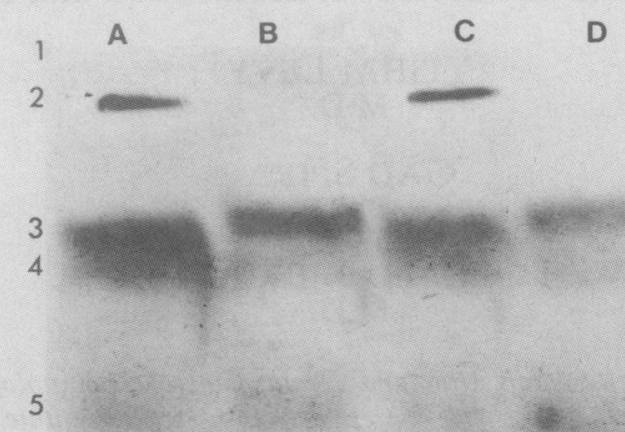

FIG. 1. Plasma lipoprotein electrophoresis of multiple myeloma patients (A, C) and healthy controls (B, D). (1, origin; 2, extra lipid band; 3, LDL; 4, VLDL; 5, HDL.)

In order to relate lipid alteration with extent of disease, patients with MM were divided into two groups (National Cancer Institute, 1973): patients in clinical Stages I and II were grouped together and compared to patients in clinical Stage III (Table 3). Plasma cholesterol and triglyceride levels were significantly lower in patients in Stage III $(P<0.05)$, whereas the concentrations of HDL-cholesterol, apoprotein A-I and apoprotein B were not significantly different between the groups. Although the $M$ protein concentration in Stage III was significantly higher than in Stages I and II, no significant correlation was found between the M protein concentration and plasma lipid pattern.

\section{Discussion}

The present study was designed to define the characteristic changes in plasma lipids in welldocumented and carefully followed populations of patients with monoclonal gammopathy. Another aim was to search for differences related to specific clinical entities as well as to tumour burden in patients with multiple myeloma. Several reports have examined the relationship between monoclonal immunoglobulin and the alteration of serum lipids. It was often assumed that $M$ protein is associated with hyperlipidaemia. Pronounced hyperlipidaemia was not evident amongst our 49 patients, suggesting that this alteration is exceptional. Our work confirms previous reports in which a low cholesterol level appears to be associated with multiple myeloma (Riesen et al., 1972; Noseda et al., 1972). To the best 


of our knowledge, it is the first time that low cholesterol levels were demonstrated in a group of patients with BMG, comparable to patients with MM.

Traditionally, the term BMG denotes the presence of monoclonal immunoglobulins in persons without evidence of myeloma, macroglobulinaemia or other related disease. The patho-physiology and the chemical significance of this abnormality has not been clearly defined. Recently, follow-up studies demonstrated a high incidence of progression of these patients to overt B cell malignancy (Kyle, 1978; Carter and Tatarsky, 1980). So far, it has not been possible to predict in which patient the abnormality will run a benign or malignant course. Further prospective studies may clarify the value of lipid abnormalities as an indicator for malignant transformation or for progression to a higher degree of malignancy amongst monoclonal gammopathies.

The mechanism underlying the hypocholesterolaemia, which was a consistent finding amongst the patients, cannot be clarified from the present data. Previous studies tried to relate the lipid alteration to the ability of the monoclonal globulin to react with lipoprotein constituents. Systematic screening of more than 200 sera containing M components for reactivity against lipoprotein disclosed that $10 \%$ gave a positive reaction with human low density lipoprotein (LDL) and very low density lipoprotein (VLDL), with no cross-reaction with HDL. Binding activity was restricted to the $F_{a b}$ fragment of the immunoglobulin molecule. It was suggested that in cases with hypolipidaemia, antibodies were directed against the protein constituent of the lipoprotein (Riesen et al., 1975).

A patient with IgA-monoclonal globulin reactive with autologous and homologous LDL and VLDL has been described (Noseda et al., 1972). Electrophoresis disclosed a relative decrease of $\alpha$-lipoprotein. An additional lipid band was observed. Treatment of the patient resulted in the disappearance of the monoclonal protein with normalization of the $\alpha$ - and $\beta$-lipoproteins and disappearance of the additional lipid band.

The presumed association between monoclonal protein and lipid abnormality led us to search for a lipid-immunoglobulin relationship. The extra lipid band appearing on acetate electrophoresis may suggest a lipid-immunoglobulin in complex, although such documentation does not mean any specific monoclonal antilipoprotein activity. The occurrence of the extra lipid band appears characteristic of those patients with low concentrations of cholesterol. Further work is being undertaken in our laboratory to determine the specific nature of this electrophoretic phenomenon.

Apoproteins have not been studied in monoclonal gammopathies. Our results demonstrate a parallel decrease in HDL-cholesterol and its major apoprotein A-I. Apoprotein B concentration, however, did not parallel the decrease in cholesterol concentrations. While LDL, the major cholesterol-carrying particle, is the major source of apoprotein $B$, the inequality in concentration of apoprotein $B$ and cholesterol indicates a possible alteration in LDL composition. The affinity of cholesterol to apoprotein B may be altered by the same mechanism leading to the other lipid alterations. Dynamic studies in patients with myelo-proliferative disorders demonstrated an increase in apoprotein B breakdown (Ginsburg, Gilbert and Gibson, 1982).

Waldenström's macroglobulinaemia deserves special consideration because of the extreme lipid alterations. The activity of IgM against various lipid constituents may explain the marked decrease in cholesterol, triglycerides and HDL-cholesterol (Killander et al., 1967; Stone and Metzger, 1968; Riesen et al., 1972).

The aim of our study was to document the occurrence of plasma lipids abnormalities in specific patient populations. The mechanism underlying the observed results can only be a matter of speculation at present: further research into the pathogenesis and significance of these alterations, in view of the biology of the different monoclonal gammopathies, is mandatory.

\section{Acknowledgments}

We wish to acknowledge with our appreciation the technical assistance of Ms. Gertrude Dankner of the Lipid Research Laboratory. This work was supported in part by the Mitchell-Copp Foundation.

\section{References}

Alexanian, R. (1980) Localized and indolent myeloma. Blood, 56, 521.

BAUDET, M.F., DACHET, C. \& BEAUMONT, J.L. (1980) Interaction between fibroblasts, lipoproteins and three anti-lipoproteins IgA kappa. Clinical and Experimental Immunology, 39, 455.

BEAUMONT, J.L. \& BEAUMONT, V. (1977) Auto-immune hyperlipidaemia. Atherosclerosis, 26, 405.

Burstein, M., Scholnick, H.R. \& Marfin, R. (1970) Rapid method for the isolation of lipoproteins from human serum by precipitation with polyanions. Journal of Lipid Research, 11, 538.

CARTER, A. \& TATARSKY, I. (1980) The pathophysiological significance of benign monoclonal gammopathy: A study of 64 cases. British Journal of Haematology, 46, 565.

Cortese, C., Lewis, B., Miller, N.E., Peyman, M.A., Rao, S.N., Slavin, B., Sule, U., TURner, P.R., UtermanN, G., Wing, A.J., WeIGHT, M. \& WoOTON, R. (1982) Myelomatosis with Type III hyperlipoproteinaemia. New England Journal of Medicine, 307, 79.

DURIE, B.G.M. \& SALMON, S.E. (1975) Clinical staging system for multiple myeloma: Correlation of measured myeloma cell mass with presenting clinical features, response to treatment and survival. Cancer, 36, 842.

FAHEY, J.L. \& MCKELVEY, E.M. (1965) Quantitative determinations of serum immunoglobulins in antibody agar plates. Journal of Immunology, 94, 84. 
GinSBURG, H., GILBERT, H.S. \& GibSON, J.C. (1982) Increased low density lipoprotein catabolism in myelo-proliferative disorders. Annals of Internal Medicine, 96, 311.

Killander, H., Killander, J., Philipson, L. \& Willen, R. (1967) A monoclonal macroglobulin complexing with lecithin. In: Nobel Symposium 3: Gamma Globulins, (ed. J. Killander), p. 359. Interscience, New York.

KYLE, R.A. (1978) Monoclonal gammopathy of undetermined significance: A natural history of 241 cases. American Journal of Medicine, 64, 814.

LAURELL, C.B. (1965) Antigen-antibody crossed electrophoresis. Analytical Biochemistry, 10, 358.

National Cancer Institute's Chronic Leukemia Myeloma TASK FORCE (1973) Proposed guidelines for protocol studies. II Plasma cell myeloma. Cancer Chemotherapy Reports, 4, 145.

Noseda, G., Riesen, W., Schlumpf, E. \& Morell, A. (1972) Hypo- $\beta$-lipoproteinaemia associated with auto-antibodies against
B-lipoprotein. European Journal of Clinical Investigation, 2, 342. RIESEN, W. \& NOSEDA, G. (1975) Antibodies against lipoproteins in man: Occurrence and biological significance. Klinische Wochenschrift, 53, 353.

Riesen, W., NosedA, G., RudRKoff, S. \& Butler, R. (1972) An IgM Waldenström with anti-phosphorylcholine specificity. Twentieth Annual Colloquium on Protides and Biological Fluids, Brugge. Roberts-Thomson, P.J., VenAbles, G.S., ONITIRI, A.C. \& LEWIS, B. (1975) Polymeric IgA myeloma, hyperlipidaemia and xanthomatosis: A further case and review. Postgraduate Medical Journal, 51, 44.

STONE, M.J. \& METZGER, H. (1968) Binding properties of Waldenström macroglobulin antibody. Journal of Biological Chemistry, 243, 5977.

(Accepted 16 November 1983) 